\title{
An upgrade of the UTR-2 radio telescope to a multifrequency radio heliograph
}

\author{
Stanislavsky A. ${ }^{1,2}$, Konovalenko A. ${ }^{1}$, Koval A. ${ }^{3}$, Volvach Ya. ${ }^{1}$ \\ ${ }^{1}$ Institute of Radio Astronomy of the NASU, Ukraine; \\ ${ }^{2}$ V.N. Karazin Kharkiv National University, Ukraine; \\ ${ }^{3}$ Institute of Space Sciences, Shandong University, China \\ E-mail: astex@ukr.net
}

Accepted:7 November 2017

\begin{abstract}
We present the broadband heliograph based on the UTR-2 radio telescope for obtaining the solar corona images in the frequency range $8-32 \mathrm{MHz}$ with the frequency resolution $4 \mathrm{kHz}$, the time resolution up to $1 \mathrm{~ms}$, and under the dynamic range about $90 \mathrm{~dB}$. The instrument provides new possibilities to measure the non-thermal radiation in an unprecedented way for a better understanding of the radio emission processes in solar corona. We describe various aspects of the instrument including its antenna system, receiver front end, digital hardware and the data acquisition. This is the lowest-frequency heliograph operating in the world. It allows us to detect radio emission from solar radio sources in the upper solar corona near frequencies of ionosphere cut-off. The instrument performance is illustrated with source maps of solar radio bursts at low frequencies during the observational campaigns of 2013 and 2015 .
\end{abstract}

(C) 2018 BBSCS RN SWS. All rights reserved

Keywords:

\section{Introduction}

Solar activity is investigated by different means. One of them, very popular, is radio observations. Coronal mass ejections (CMEs), shock waves, electron beams, flares and many others lead to solar radio bursts. Their study permits us to understand solar activity phenomena remotely, since the study of the phenomena in situ is impossible. Solar corona is a unique place. On the one hand, the corona is an origin of solar bursts, but their radio emission propagates through the plasma of corona that has an index of refraction, scattering and so on. All the effects should be taken into account, but this is not a simple task. The corona changes due to many complex processes (flares, electron beams, shock waves and others). Of course, the bursts observed by groundbased instruments are gleams of such processes. If temporal and frequency properties of solar bursts were examined fairly well, then their source imaging studies remain still limited, especially at low frequencies. This is because the number of radio telescopes suitable to getting a radio image of the Sun at low frequencies is very small. Many years ago the low-frequency facilities were operated at Culgoora (Labrum, 1985) in Australia at $40-320 \mathrm{MHz}$ and at Clark Lake (Erickson et al., 1974) in the USA at 20-110 MHz. Unfortunately, they were taken out of service. Now there is a revival of lowfrequency radio astronomy. In this regard, it should be noted the large-scale wide band project LOFAR as well as antenna arrays LWA (Taylor et al., 2012) and MWA (Tingay et al., 2013) and the Gauribidanur radio heliograph in the frequency band $30-110 \mathrm{MHz}$ at a few discrete frequencies (Ramesh et al., 1998). The radio heliograph based on the UTR-2 antenna array is also an appropriate instrument for above-mentioned goals. Its advantage lies in the fact that it is the lowestfrequency heliograph operating in the world. The aim of this work is to present the characteristics of this tool as well as its applications in recent solar researches.

\section{Heliograph Design}

The main performance of the UTR-2 heliograph is determined by the antenna characteristics of UTR-2 (Konovalenko et al., 2016). The broadband T-shaped radio telescope UTR-2 includes three antenna arrays: "North", "South" and "West". Each array has four sections. The larger arm "North-South" has 1800 meters along meridian and consists of 1440 wideband dipoles (6 rows by 240 dipoles). The arm "West" spreads over 900 meters along parallel and includes 600 dipoles (100 rows by 6 dipoles). All the dipoles in the arrays are oriented in the east-west direction and receive only linear polarization. It should be noticed that the use of wide-band dipoles makes it possible to carry out radio observations from $8 \mathrm{MHz}$ to $32 \mathrm{MHz}$. At the zenith the telescope beam has the angular resolution about $25^{\prime} \times 25^{\prime}$ at $25 \mathrm{MHz}$, and the effective area of the UTR-2 antenna system is about $140000 \mathrm{~m} 2$. The heliograph image is obtained from signals received with help of the fast scanning of a sky area around the Sun by the pencil-shaped beam of the UTR-2 antenna pattern. In this case the beam takes five consecutive positions along $V$ and eight positions along $U$ coordinate in UVplane. The complete field of view is a rectangular matrix consisting of 40 elements (pixels). The matrix image contains 5 rows and 8 columns spaced along declination and hour angle, respectively. Basically (but not necessarily) the heliograph uses a serial regime, where the output signals from five beams are recorded one after the other, and the five beams of the antenna array pattern move on sky (see more details in Stanislavsky et al., 2011). The angular size of the heliograph image at $25 \mathrm{MHz}$ is about $3.3^{\circ} \times 2.5^{\circ}$ along hour angle and declination at the zenith direction, respectively. The radio telescope UTR-2 provides the Sun tracking in sky so that the center in the field of view coincides with the solar disk center, while the movement of the pencil-shaped beam in the heliograph frame is performed due to a special 


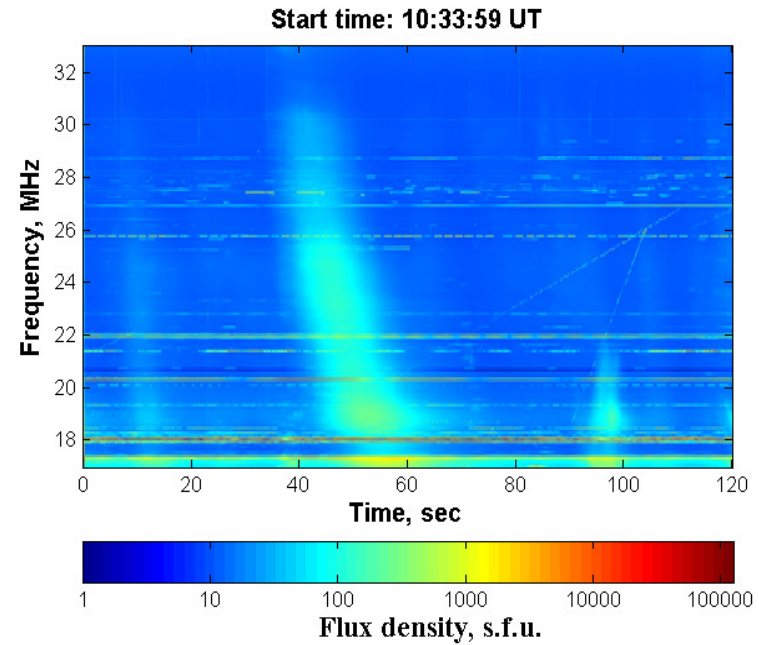

Figure 1: Illustrative dynamic spectrum of a type III burst obtained from the UTR-2 observations on 9 April of 2013. Bright horizontal lines on the dynamic spectrum show intensive radio interferences from broadcast radio stations, and some inclined lines are due to radio emission of ionosphere stations. The absolute flux-density determination was done in the traditional manner, i.e. spectral density of received radio emission was found by the comparison with the well-known spectral density of a reference noise source, and the values of the antenna effective area were tabulated. Recall also that 1 s.f.u. $=10^{-22} \mathrm{~W} /\left(\mathrm{Hz} \mathrm{m}^{2}\right)$.

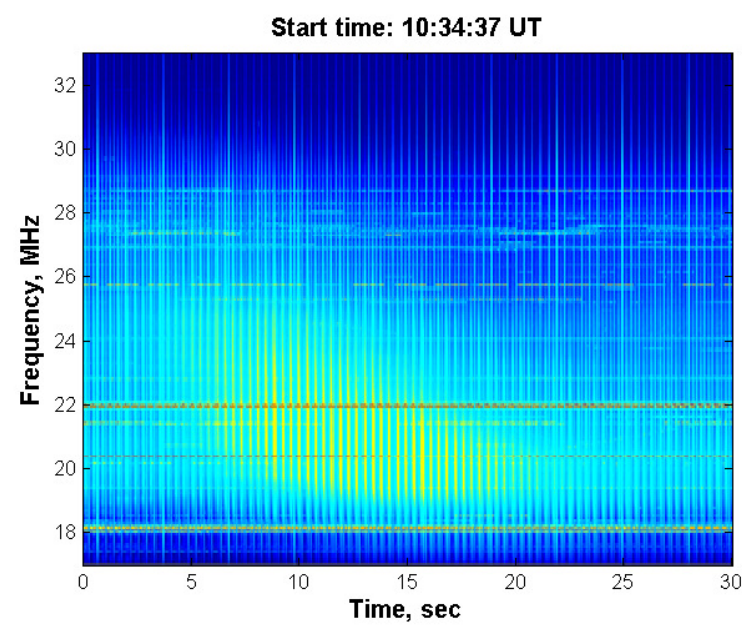

Figure 2: Heliogram records of the type III burst shown in Figure 1. Bright vertical stripes indicate marker signals. The white circle indicates the solar disk. Each frequency shot is resized by a factor of 10 , i.e., it includes the matrix of $50 \times 80$ pixels.

fast phase shifter. For shaping pencil beams, the signal from the "West" antenna output is multiplied by signals from outputs of the "North-South" antenna five beams with help of the two-channel digital receivingrecording device (named DSPZ from "Digital SpectroPolarimeter, type Z"), working in the correlation regime (Zakharenko et al., 2016). Consequently, the system forming beams of the UTR-2 produces five pencilshaped beams. In the serial regime the output signals from five beams are recorded one after the other by one DSPZ. In the end of each beam scanning cycle, instead of antennas, the auxiliary noise generator, which helps forming markers on the heliogram, is connected to the receiving-recording device. Such markers allow easily recovering heliogram images from the serial DSPZ records. The extra phase shift module is only connected with outputs of West array sections. Therefore, the heliograph mode does not interfere with taking signals from North-South sections for studying dynamic spectra of received solar bursts.

Using the heliograph, we can observe both dynamic spectra of solar bursts and their heliograms. The heliograph images are obtained from signals received due to the fast scanning of a given sky area (including the Sun) by the pencil-shaped beam of the UTR-2 antenna pattern. The heliograms allow us to recognize the source positions of solar burst sources at any fixed frequency and to estimate the source sizes along $U$ and $V$.

\section{Data}

On 2013 April 9-11 we observed many type III radio and IIIb-III bursts by using both spectral (Figure 1) and heliographic (Figure 2) regimes at 16.5-32 MHz. Solar radio signals of each beam are recorded in the form of dynamic spectra with both high temporal ( 2.48 ms) and top spectral $(\sim 4 \mathrm{kHz})$ resolutions. The rate of output heliograph was one image per 3 seconds (Figure 3). This permitted us to study two-dimensional spatial structures of burst sources in dependence of frequency and time.

The solar III type bursts are the most numerous events filling the low-frequency band (<100 MHz). In decameter wavelengths the type III bursts have typically drift rates about - (2-4) MHz/s. Such radio emission is associated with beams of fast electrons moving outward into interplanetary space along open magnetic field lines (see more details, for example, in Reid and Ratcliffe, 2014). Durations of these bursts can be up to $20 \mathrm{~s}$, while the most are found in the range within 4-10 $\mathrm{s}$ in the frequency band 10-30 MHz. The energetic characteristics of decameter type III bursts is that their brightness temperatures can achieve to $10^{8}-$ $10^{10} \mathrm{~K}$ and more. Until recently, the study of type III bursts is restricted by the conventional analysis of frequency-time characteristics from dynamic spectra. The solar burst sources have not been imaged extensively because of the instrumental limitations of previous radio telescopes. On 2013 February 28, several hours after the launch of two CMEs, over 30 radio bursts of type III were observed by LOFAR tiedarray beams imaging and spectroscopy at 30-90 $\mathrm{MHz}$. The study has discovered that the type III bursts occurred at altitudes in excess of values predicted by ID radial electron density models, and the non-radial high altitude type III bursts were associated with the expanding flank of a coronal mass ejection (CME). In this connection it should be noticed that our heliograph observations can provide the research by spatially-resolved data for the solar bursts (generated in upper corona) at 8-32 $\mathrm{MHz}$.

During our radio observations on 2013 April 9-11, many sunspots were found on solar disk. Among them were both unipolar and complex sunspot groups. Moreover, most of them were active in the form of solar flares of $C$ and $M$ classes. This explains a poor character (with rare exceptions) of CMEs observed in that time (see more details in https://cdaw.gsfc.nasa.gov/CME list/UNIVERSAL/2013 04/univ2013_04.html). 


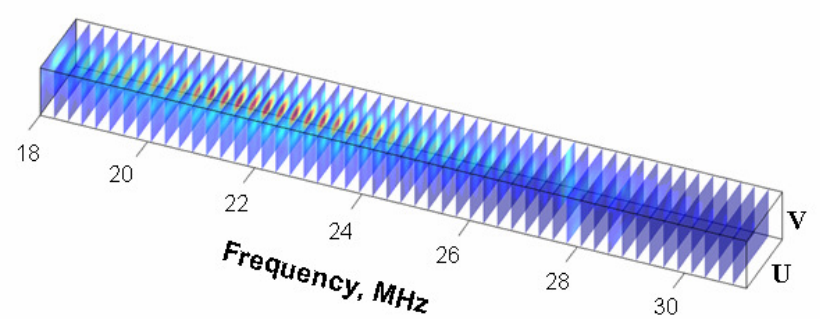

Figure 3: Heliogram cube of the type III burst. This picture is updated every 3 seconds.

Another observational session was carried out on 2015 September 14. In the heliographic observations we have detected the solar drift pair bursts. For brevity, let us call them simply the drift pairs (DPs). Most of them were really pairs on dynamic spectra, but we have also noticed a few vertical DPs as well as several single and multiple bursts like DPs. The current state of spectral research of DPs is presented in the recent paper of Stanislavsky et al. (2017). However, as for the mapping of DP sources, one can mention only the long-standing paper of Suzuki and Gary (1979). The radio imaging of DPs were carried out at a single frequency (at $43 \mathrm{MHz}$ ), and new heliographic observations are very relevant. Eventually we have identified 21 DP bursts in which 15 were forward, and the others were reverse in frequency drift. Moreover, two S-bursts have been seen as well. Solar activity was very weak, no flares of $C$ class and higher. Several solar flares of $C$ class were observed during the next day (from NOAA AR 12415 with S22E30).

\section{Results}

Using the UTR-2 heliograph, we observed the evolution of angular structure of burst sources in about 2-3 radii from the center of the Sun.

\section{a) Solar type III and IIIb-III bursts}

At these heights the angular dimensions of type III burst sources reach about one solar radius and even more. Basically, the heliogram analysis was performed for the type III and IIIb-III bursts. By data processing the serial records were converted into a set of twodimensional images. Consequently, the three dimensional angular structure evolution of the type III solar burst sources (UV-plane frames at selected frequencies in dependence of time) was obtained (see, as an example, Figure 3). The peak-in-time evolution of such bursts from frame to frame permits us to detect source-motion directions for these bursts. Unfortunately, from the heliograph data we were unable to clearly notice the non-radial high altitude type III bursts associated with CMEs. Probably, this is connected with the fact that the CMEs were too weak, although it is possible that they moved from the Sun without significant impact on the type III burst sources. Nevertheless, this remark does not mean that the source motion for solar bursts cannot be detected by the UTR-2 radio heliograph in other observations. For these purposes, there must be suitable conditions. Recently, the UTR-2 heliographic observations of stationary type IV radio emission, done on 2014 September 6, at 16.5-32 MHz have been presented by Koval et al. (2014). Their analysis earnestly shows that the dynamical motion of radio sources, responsible for this event, manifests the loop response to the CME disturbance.

\section{b) Drift pair bursts}

Using the spectral data, the frequency drift rates of DPs were determined in the same manner as in the paper of Volvach et al. (2016). Such events is shown in Figure 4(a). In each frequency channel of the digital records (within the frequency band, where the chosen DP was detected) for each component of DPs we found the "center" of the intensity hump. The peak evolution of such bursts in the frequency-time plane was fitted to the power function. Then the frequency drift rate is written as

$$
\dot{f}(f)=K f^{v},
$$

where $K$ and $v$ are the constants which can be determined from the above-mentioned fitting procedure. As the number of DPs, identified by us in the observational session, was relatively small, their properties are collected in Table 1. The sign of $\mathrm{K}$ indicates whether the frequency drift rate is positive (reverse DPs) or negative (forward DPs).

Table 1. Frequency drift rate of the DPs observed on 14 September of 2015. Here $K>0$ corresponds to reverse DPs whereas $K<0$ relates to forward DPs. The index (either 1 or 2) characterizes one or another component in each DP. The right column $\Delta f$ is the full frequency band occupied by each burst in $\mathrm{MHz}$.

\begin{tabular}{|c|c|c|c|c|}
\hline$K_{1}$ & $V_{1}$ & $K_{2}$ & $V_{2}$ & $\Delta f$ \\
\hline$-0,044$ & 1,45 & $-0,008$ & 2,01 & 4,65 \\
\hline$-0,001$ & 2,89 & $-0,00036$ & 2,52 & 2,99 \\
\hline$-0,0011$ & 2,22 & $-0,0012$ & 2,22 & 3,72 \\
\hline$-0,0011$ & 2,13 & $-0,0018$ & 1,95 & 5,93 \\
\hline$-0,0006$ & 2,73 & $-0,000074$ & 2,98 & 4,95 \\
\hline$-0,0012$ & 2,11 & $-0,0016$ & 2,03 & 7,59 \\
\hline$-0,0001$ & 3,07 & $-0,014$ & 1,26 & 1,62 \\
\hline$-7,68 \mathrm{E}-09$ & 5,3164 & $-0,0012$ & 2,1 & 1,81 \\
\hline$-0,0029$ & 1,9127 & $-0,000013$ & 3,68 & 1,67 \\
\hline$-0,0016$ & 2,1214 & $-0,0014$ & 2,13 & 3,77 \\
\hline$-9,85 E-05$ & 2,8652 & $-0,0000079$ & 3,66 & 1,67 \\
\hline -7,9E-06 & 3,6592 & $-0,00068$ & 2,31 & 4,36 \\
\hline$-0,002$ & 1,9901 & $-0,00072$ & 2,33 & 4,11 \\
\hline$-0,0012$ & 2,0617 & $-0,0014$ & 2 & 9,75 \\
\hline$-0,00085$ & 2,2731 & $-0,00081$ & 2,25 & 3,67 \\
\hline 0,47 & 0,98 & 0,05 & 0,97 & 1,67 \\
\hline 0,058 & 0,98 & 0,07 & 0,96 & 1,86 \\
\hline 0,064 & 0,98 & 0,049 & 0,98 & 1,67 \\
\hline 0,058 & 0,98 & 0,05 & 0,97 & 1,67 \\
\hline 0,046 & 0,98 & 0,056 & 0,98 & 1,81 \\
\hline 0,047 & 0,98 & 0,057 & 0,97 & 1,81 \\
\hline
\end{tabular}

The flux of the DP bursts was about some tens to one hundred s.f.u. Not every DP, found us in dynamic spectra, was clearly distinguishable on heliograms. The point is that the sensitivity of heliographic measurements is weaker than in spectral records. The full frequency bandwidth occupied by the DPs varied from $1.62 \mathrm{MHz}$ to $9.75 \mathrm{MHz}$. Moreover, the number of forward DPs was more than the number of reverse ones. The same behavior was observed on July 10-12, 2015. During the observations (Volvach et al., 2016), the occurrence of forward DPs is more preferable at lower frequencies of the decameter range in comparison with reverse DPs generated more likely at meter wavelengths. Each DP had the duration equal to about 1-2 $s$, and the time delay between components was $1.5-2.5 \mathrm{~s}$. 


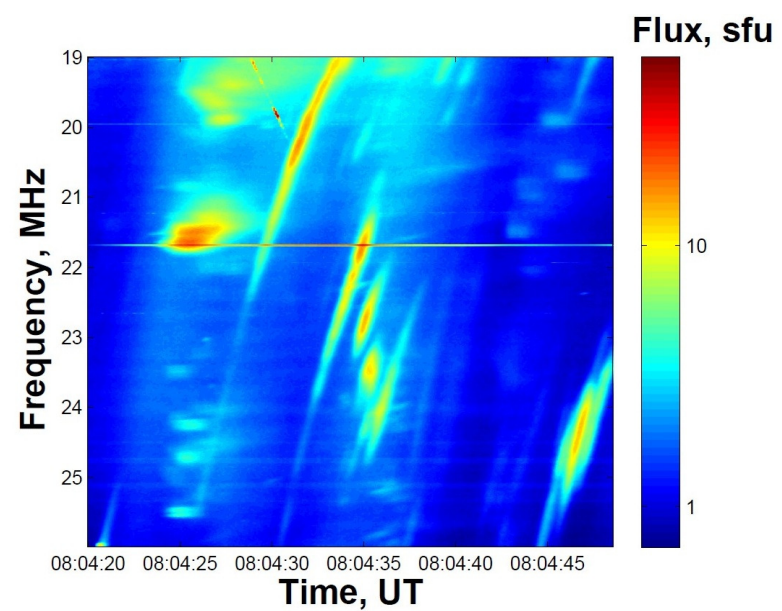

(a)

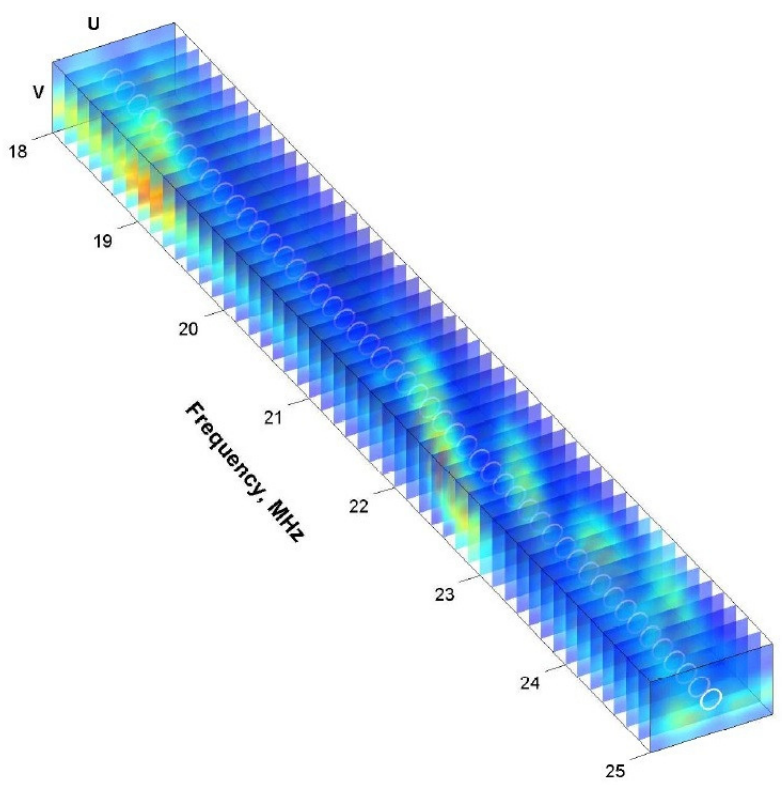

(b)

Figure 4: Dynamic spectrum with DPs and the like (a) with their heliogram at 08:04:35 UT (b). In the heliographic frames the radio emission increases within $21-25 \mathrm{MHz}$ in the form of "outstretched clouds". The intensity value is expressed in relative units.

Using the heliographic data, we have explored also spatial properties of the DP (and the like) sources at 8$32 \mathrm{MHz}$ by recording one frame every $3 \mathrm{~s}$. For the studies of short bursts, this rate is small. But to make it higher, we would have to go into the mode of DSP waveform, which is much more difficult in signal processing. To observe changes of source positions in the solar corona, there is no necessity to account for the entire abundance of frequency channels. Therefore, we used about 50 equidistant frequencies (or even less) in each frame. An example of such a heliogram is shown in Figure 4 (b). On the heliogram one can see short components of DPs, separated in time, in the frequency band 21-25 MHz about 08:04:35 UT. From the heliographic observations of DPs, we have found that the angular sizes of DPs were less than the UTR-2 beam size. In particular, they were less than $25^{\prime}$ at $25 \mathrm{MHz}$. Nevertheless, the heliograms allowed us to recognize the source positions of different DPs (and the like) at fixed frequencies. This relates to both forward and reverse DPs. Interestingly, the angular sizes of the storm type III burst sources observed together the DPs were also less than the UTR-2 beam size. As the angular sizes of ordinary type III bursts are determined by the scattering of radio emission due to inhomogeneity in the solar corona, smaller sizes of DP sources land related bursts of type III) indicate in favor of smallerscale inhomogeneity above the emitting sources. By the way, this feature was accompanied by weak solar activity. All this is consistent with the results of Suzuki and Gary (1979) obtained at a higher frequency. To provide the angular selection of DP component sources in this frequency range, we are going to perform special interferometer measurements in prospect.

\section{Conclusions}

The angular dimensions of sources of solar radio bursts are still not explored intensively at low frequencies. The two-dimensional heliograph, based on the UTR-2, already gives new information about the space structure of solar burst sources within 8-32 MHz. In the future it would be interesting to provide joint solar observations by our instrument with the LOFAR tied-array beams imaging. They together could perfectly complement each other, operating in various (but close to each other) low-frequency bands.

\section{Acknowledgements}

We would like to thank the GOES, STEREO and $\mathrm{SOHO}$ teams for developing and operating the instruments and we are grateful for their open data policy. This research was partially supported by Research Grant $0117 \cup 002395$ from the National Academy of Sciences of Ukraine.

\section{References}

Erickson, W.C., and Fisher, J.R.: 1974, Radio Science, 9, 387. doi:10.1029/RS009i003p00387

Konovalenko, A., Sodin, L., Zakharenko, V., et al.: 2016, Experim. Astron. 42, 11. doi:10.1007/s10686-016-9498-x

Koval, A.A., Stanislavsky, A.A., Chen, Y., Feng, Sh., Konovalenko, A.A., and Volvach Ya.S.: 2016, Astrophys. J. 826, A125. doi: $10.3847 / 0004-637 X / 826 / 2 / 125$

Labrum, N.R.: 1985, in McLean D.J. and Labrum N.R. (eds.), "Solar radiophysics: Studies of emission from the sun at metre wavelengths", Cambridge University Press, New York, p. 155.

Morosan, D.E. ., Gallagher, P. T., Zucca, P., et al.: 2014, Astron. Astrophys. 568, id. A67. doi:10.1051/0004-6361/201423936

Ramesh, R., Subramanian, K.R., Sundararajan, M.S., and Sastry, Ch.V.: 1998 , Solar Phys . 181, 439. doi:10.1023/A:1005075003370

Reid, H.A.S., and Ratcliffe, H.: 2014, Res. Astron. Astrophys. 14, 773. doi:10.1088/1674-4527/14/7/003

Stanislavsky, A.A., Koval, A.A., Konovalenko, A.A., and Abranin, E.P.: 2011, https://arxiv.org/abs/1112.1044.

Stanislavsky, A.A., Konovalenko A.A., and Volvach Ya.S.: 2017, Res. Astron. Astrophys. 17, id. 097. doi: 10.1088/1674$4527 / 17 / 9 / 97$

Suzuki, S., and Gary, D.E.: 1979, Astronomical Society of Australia $3,379$.

Taylor, G.B., Ellingson, S.W., Kassim, N.E., et al.: 2012, J. Astron. Instrum. 01, id.1250004. doi: 10.1142/S2251171712500043

Tingay, S.J., Goeke, R., Bowman, J.D., et al.: 2013, Publ. Astron. Soc. Austral. 30, id.e007. doi:10.1017/pasa.2012.007

Volvach, Ya.S., Stanislavsky A.A., Konovalenko A.A., Koval A.A., and Dorovskyy V.V.: 2016, Advanced in Astronomy and Space Physics 6, 24. doi: 10.17721/2227-1481.6.24-27

Zakharenko, V., Konovalenko, A., Zarka, P., et al.: 2016, J. Astron. Instrum. 05, id. 1641010. doi: $10.1142 /$ S2251171716410105 